\title{
Poecilancistrium caryophyllum (Diesing, 1850) (Cestoda, Trypanorhyncha), parasito de Macrodon ancylodon (Bloch \& Schneider, 1801) do litoral Norte do Brasil
}

\author{
Poecilancistrium caryophyllum (Diesing, 1850) (Cestoda, Trypanorhyncha), parasite of Macrodon ancylodon
} (Bloch \& Schneider, 1801) from the Northern littoral of Brazil

Silvio Abner Lameira de Oliveira ${ }^{1}$; Sérgio Carmona de São Clemente; Raimundo Nonato Moraes Benigno ${ }^{3}$; Marcelo Knoff ${ }^{4}$

\author{
${ }^{1}$ Universidade Federal do Pará - UFPA \\ ${ }^{2}$ Faculdade de Veterinária, Universidade Federal Fluminense - UFF \\ ${ }^{3}$ Universidade Federal Rural da Amazônia - UFRA \\ ${ }^{4}$ Laboratório de Helmintos Parasitos de Vertebrados, Instituto Oswaldo Cruz, Fundação Oswaldo Cruz
}

Recebido em 16 Abril de 2009

Aceito em 1 de Julho de 2009

\section{Resumo}

O objetivo desta pesquisa foi registrar a presença de cestoides Trypanorhyncha, suas prevalências e intensidades de infecção, na musculatura e serosa abdominal parietal de 50 exemplares de Macrodon ancylodon, capturados no litoral Norte do Brasil e beneficiados em Belém, Estado do Pará, durante o período de junho de 2004 a janeiro de 2005. A musculatura e serosa abdominal dos peixes foram examinadas em mesa de inspeção candling table após o filetamento das amostras. Oito peixes encontravam-se parasitados por plerocercoides da espécie Poecilancistrium caryophyllum, com prevalência de $16 \%$ e intensidade média de infecção de um plerocerco por peixe, tendo como único sítio de infecçáo a musculatura. A espécie Poecilancistrium caryophyllum é, pela primeira vez, registrada para $M$. ancylodon.

Palavras-chave: Trypanorhyncha, Poecilancistrium caryophyllum, Macrodon ancylodon, Brasil.

\begin{abstract}
The aim of this research was to report the presence of Trypanorhyncha cestodes, their prevalence and mean intensity in the muscles musculature and abdominal serose in commercial fish processed in Belém, Pará State. Fifty specimens of M. ancylodon were captured in the North Coast of Brazil. The musculature and adjacent abdominal serose of fish were analized in a "candling table" after filleted. Eight specimens were parasitized by plerocercoids of Poecilancistrium caryophyllum, with a prevalence of $16 \%$ and mean intensity of infection of one plerocercus per fish, varying from the unique site of infection was the musculature. The species $P$. caryophyllum is reported by the first time on M. ancylodon.
\end{abstract}

Keywords: Trypanorhyncha, Poecilancistrium caryophyllum, Macrodon ancylodon, Brazil.

O pescado destinado ao consumo humano deve ser alvo de maior preocupação por parte dos serviços de fiscalização sanitária, pois algumas enfermidades de peixes têm caráter zoonótico, como é o caso do parasitismo por nematoides anisaquídeos, por trematódeos digenéticos e por cestoides difilobotrídeos (THATCHER; BRITES NETO, 1994). Alguns parasitos, entretanto, mesmo não sendo transmissíveis ao homem, adquirem importância quando causam repugnância em relação à aparência da carne parasitada

\footnotetext{
*Autor para correspondência: Sérgio Carmona de São Clemente Faculdade de Veterinária, Universidade Federal Fluminense - UFF, Rua Vital Brazil, 64, CEP 24230-340, Niterói - RJ, Brasil; e-mail: scsc@vm.uff.br
}

pelos helmintos. Nesse contexto, situam-se os cestoides da ordem Trypanorhyncha, objetivo de diversos estudos em peixes do litoral brasileiro (CARVAJAL; REGO, 1983, 1985; SÃO CLEMENTE, 1986, 1987; AMATO; SÃO CLEMENTE; OLIVEIRA, 1990; SÃO CLEMENTE; COELHO; SERRA-FREIRE, 1991; 1993; SÃO CLEMENTE; LIMA; UCHOA, 1995; SÃO CLEMENTE; SILVA; GOTTSCHALK, 1997; 2004; PEREIRA JR., 1993; PALM, 1997; SILVA; SÃO CLEMENTE, 2001; SABAS; LUQUE, 2003; PEREIRA JR.; BOEGER, 2005).

Os cestoides adultos da ordem Trypanorhyncha têm como hospedeiros definitivos os peixes elasmobrânquios, e suas formas larvais parasitam várias espécies de teleósteos e de invertebrados marinhos diversos (CAMPBELL; BEVERIDGE, 1994). 
A pescada-gó, ou pescada-foguete (Macrodon ancylodon), é um peixe costeiro com hábito demersal, da família Sciaenidae, sendo sua distribuição restrita à parte ocidental do oceano Atlântico, ocorrendo da Venezuela até a Argentina (SANTOS; SCHNEIDER; SAMPAIO, 2003).

Sabas e Luque (2003) estudando quantitativamente os componentes e a estrutura das comunidades parasitárias de M. ancylodon do litoral do Estado do Rio de Janeiro, encontraram parasitismo por larvas de três espécies de Trypanorhyncha: Callitetrarhynchus gracilis, Nybelinia sp. e Progrillotia dollfusi (mesentério). Também nesse scianídeo capturado no litoral Sul do Brasil, Pereira Jr. e Böeger (2005) identificaram larvas de Trypanorhyncha das espécies C. gracilis (rim e cavidade celomática), Dasyrhynchus pacificus (rim, cavidades celomática e pericárdica), e $P$. dollfusi (cavidade celomática).

No Brasil há poucos registros que assinalam índices de parasitismo por larvas de cestoides Trypanorhyncha restritos à musculatura, porque esse grupo de helmintos é encontrado com frequência na cavidade abdominal e serosas de órgãos internos de peixes teleósteos (AMATO; SÃO CLEMENTE; OLIVEIRA, 1990; SÃO CLEMENTE; COELHO; SERRA-FREIRE, 1991; 1993; SÃO CLEMENTE; LIMA; UCHOA, 1995; SÃO CLEMENTE; SILVA; GOTTSCHALK, 1997; 2007; PALM, 1997).

O presente trabalho teve como objetivo estudar a biota de cestoides Trypanorhyncha, parasitos da musculatura de $M$. ancylodon capturados no litoral Norte do Brasil.

No período compreendido entre junho de 2004 e janeiro de 2005, foram adquiridos 50 exemplares de pescada-gó, Macrodon ancylodon, com 18 a $30 \mathrm{~cm}$ de comprimento padrão, separados em três classes de tamanho: classe 1, sete espécimes: menores do que $20 \mathrm{~cm}$; classe 2, 21 espécimes, medindo de 21 a $25 \mathrm{~cm}$; e classe 3, 22 espécimes, medindo de 26 a $30 \mathrm{~cm}$, capturados em águas litorâneas dos Estados do Pará e Amapá.

Os peixes foram adquiridos na linha de inspeção já eviscerados e mensurados em seu comprimento padrão, e os filés retirados ao longo de todo o comprimento do peixe, por meio de incisão próxima aos opérculos até a inserção da nadadeira caudal, preservando-se a serosa abdominal parietal. Os filés foram inspecionados em mesa de inspeção do tipo candling table, que, por transparência, permite a visualização dos cistos de parasitos entre os tecidos examinados.

Os plerocercos coletados e após liberados dos blastocistos foram fixados em álcool, formol e ácido acético (AFA), clarificados em creosoto, corados com hematoxilina, e montados entre lâminas e lamínulas em bálsamo do Canadá. Para determinação taxônomica dos cestoides foram utilizadas as determinaçóes de Dollfus (1942) e Campbell e Beveridge (1994). Espécime representativo da espécie encontrada foi depositado na Coleção Helmintológica do Instituto Oswaldo Cruz (CHIOC), Estado do Rio de Janeiro, Brasil. Os índices parasitários de prevalência e intensidade média de infecção foram calculados segundo Bush et al. (1997).

Dos peixes examinados oito apresentaram-se parasitados por Poecilancistrium caryophyllum, com prevalência de $16 \%$ e intensidade média parasitária de um plerocerco por peixe. $\mathrm{O}$ único sítio de infecção observado foi a musculatura dorso-lateral. Um espécime representativo, preservado em álcool $70^{\circ} \mathrm{GL}$, foi depositado na $\mathrm{CHIOC}$ sob o número 35.650. A classe de peixes de menor tamanho $(<20 \mathrm{~cm})$ não apresentou infecção, e os peixes pertencentes à classe de maior tamanho (26-30) contribuíram com o maior número de blastocistos recuperados (23,8\%) (Tabela 1). A análise pelo Teste Kruskal-Wallis demonstrou que náo houve correlação entre o grau de parasitismo e o tamanho dos peixes.

Em relação ao parasitismo de Trypanorhyncha em $M$. ancylodon, os dados obtidos por Sabas e Luque (2003) e Pereira Jr. e Böeger (2005) permitem algumas comparaçôes com o presente estudo. Embora tenham encontrado outras espécies de Trypanorhyncha parasitando $M$. ancylodon, no presente trabalho somente P. caryophyllum foi observado. Então os resultados daqueles autores demonstraram infecçóes mistas por três espécies de cestoides e ausência de parasitismo muscular.

Nesta observação, a infecção era por uma única espécie e somente nos músculos. Os índices de infecção pelas espécies de Trypanorhyncha revelados neste estudo, para a mesma espécie de peixe, demonstraram que o valor mais próximo ao observado (16\%) foi o registrado por Sabas e Luque (2003), de 11,4\% para Nybelinia sp. As outras espécies encontradas pelos referidos autores, Progrillotia dollfusi e Callitetrarhynchus gracilis apresentaram índices de 48,6 e 3\%, respectivamente. Para todas as espécies de cestoides estudadas, as intensidades médias parasitárias variaram de 1 a 4,5 espécimes por peixe, semelhante ao resultado desta trabalho que foi de um parasito.

No estudo de Pereira Jr. e Böeger (2005), a prevalência de parasitismo apresentada por $P$. dollfusi foi superior $(96,67 \%)$, e pelas espécies Dasyrhynchus pacificus (5\%) e C. gracilis (1,67\%) foram inferiores. Com exceção de Progrillotia dollfusi, que apresentou intensidade média igual a 792,21, as outras espécies encontradas por esses autores apresentaram a mesma intensidade média baixa, igual a um parasito por peixe, similar à apresentada neste relato para P. caryophyllum.

Não existia até o presente estudo nenhum registro por infecção de P. caryophyllum em $M$. ancylodon, apesar de este cestoide ter os peixes scianídeos como seus principais hospedeiros (OVERSTREET, 1977). A prevalência de $P$. caryophyllum em peixes da família Sciaenidae do

Tabela 1. Relação entre prevalência (P) e intensidade média (IM) de infecção de Poecilancistrium caryophyllum (Cestoda: Trypanorhyncha) parasitando a musculatura de Macrodon ancylodon e as classes de tamanho dos peixes (comprimento padrão) coletados, entre Junho de 2004 e Janeiro de 2005, no município de Belém, Estado do Pará, Brasil.

\begin{tabular}{ccc}
\hline Tamanho dos peixes $(\mathbf{c m})$ & $\mathbf{P}(\%)$ & IM \\
\hline$<20$ & 0 & 0 \\
$21-25$ & 13,6 & 1 \\
$26-30$ & 23,8 & 1 \\
\hline
\end{tabular}


litoral do Estado do Mississipi (E.U.A) atinge 49\%, índice superior ao encontrado na presente pesquisa (OVERSTREET, 1977). Por outro lado, São Clemente (1986) encontrou baixa prevalência $(0,9 \%)$ em corvinas, Micropogonias furnieri, capturadas no litoral do Estado do Rio de Janeiro. Contudo, esses trabalhos estão de acordo quanto à localização preferencial do parasitismo na musculatura, especialmente a dorso-lateral, negatividade de infecção nos peixes menores e baixa intensidade média de parasitismo. Nesse aspecto, os dados obtidos por Overstreet (1977) de 2,3 larvas por peixe foram próximos aos obtidos neste trabalho, resultado também semelhante ao observado por São Clemente (1986).

A maior prevalência parasitária apresentada em peixes de maior tamanho está de acordo com os resultados obtidos por diversos autores (OVERSTREET, 1977; SÃO CLEMENTE, 1986, 1987; AMATO; SÃO CLEMENTE; OLIVEIRA, 1990; SÃO CLEMENTE; COELHO; SERRA-FREIRE, 1991; SÃO CLEMENTE; LIMA; UCHOA, 1995; SÃO CLEMENTE; SILVA; GOTTSCHALK, 1997) embora,sem correlação estatística neste relato. Necessário ressaltar que generalizaçóes relacionando a influência do tamanho dos peixes sobre a composiçáo qualitativa e quantitativa de infracomunidades parasitárias devem ser evitadas, como já postulado por Saad-Fares e Combes (1992).

\section{Referências}

AMATO, J. R. F.; SÃO Clemente, S. C.; OliVEIRA, G. A. Tentacularia coryphaenae Bosc, 1801 (Eucestoda: Trypanorhyncha) in the inspection and technology of the skipjack tuna, Katsuwonus pelamis (L.) (Pisces: Scombridae). Atlântica, v. 12, n. 1, p. 73-77, 1990.

BUSH, A. O. et al. Parasitology meets ecology on its own terms: Margolis et al. Revisited. Journal of Parasitology, v. 83, n. 4, p. $575-583,1997$.

CAMPBELL, R. A.; BEVERIGDE, I. Order Trypanorhyncha. In: KHALIL, L. F.; JONES, A.; BRAY, R. A. Keys to the Cestode Parasites of Vertebrates. St. Albans: CAB International, 1994. p. 51-148.

CARVAJAl, J.; REGO, A. A. Progrillotia dollfusi sp. n. (Cestoda: Trypanorhyncha) parasito de pescada do litoral brasileiro. Memórias do Instituto Oswaldo Cruz, v. 78, n. 2, p. 231-234, 1983.

CARVAJAL, J.; REGO, A. A. Critical studies on the genus Callitetrarhynchus (Cestoda: Trypanorhyncha) with recognition of Rhynchobothrium speciosum Linton, 1897 as a valid species of the genus Callitetrarhynchus. Systematic Parasitology, v. 7, n. 3, p. 161-167, 1985.

DOLLFUS, R. P. Études critiques sur les tétrarhynques du Muséum de Paris. Archives du Museum National d'Histoire Naturelle, v. 19, n. 6, p. 1-466, 1942.

OVERSTREet, R. M. Poecilancistrium caryophyllum and other Trypanorhynch cestode plerocercoids from the musculature of Cynoscion nebulosus and other sciaenid fishes in the Gulf of Mexico. Journal of Parasitology, v. 63, n. 5, p. 780-789, 1977.

PALM, H. Trypanorhynch cestode of commercial fishes from northeast brazilian coastal waters. Memórias do Instituto Oswaldo Cruz, v. 92, n. 1, p. 69-79, 1997.

PEREIRA Jr., J. O complexo de espécies de Trypanorhyncha (Cestoda), em corvinas Micropogonias furnieri do Rio Grande do Sul. Arquivos da Faculdade de Veterinária UFRGS, v. 21, n. 1, p. 58-70, 1993.
PEREIRA Jr, J.; BOEGER, W. A. Larval tapeworms (Platyhelminthes, Cestoda) from sciaenid fishes of the southern coast of Brazil. Zoosystema, v. 27 , n. 1, p. 5-25, 2005.

SAAD-FARES, A.; COMBES, C. Abundance/host size relationships in a fish trematode community. Journal of Helminthology, v. 66, n. 3, p. $187-192,1992$.

SABAS, C. S. S.; LUQUE, J. L. Metazoan parasites of weakfish, Cynoscion guatucupa and Macrodon acylodon (Osteichthyes: Sciaenidae), from the coastal zone of the State of Rio de Janeiro, Brazil. Revista Brasileira de Parasitologia Veterinária, v. 12, n. 4, p. 171-178, 2003.

SANTOS, S.; SCHNEIDER, H.; SAMPAIO, I. Genetic differentiation of Macrodon ancylodon (Sciaenidae, Perciformes) populations in Atlantic coastal waters of South America as revealed by mtDNA analysis. Genetics and Molecular Biology, v. 26, n. 2, p. 151-161, 2003.

SÃO CLEMENTE, S. C. Prevalência e intensidade média de infecção de plerocercos de Trypanorhyncha parasitando Corvina Micropogonias furnieri (Desmarest) no litoral do Estado do Rio de Janeiro. Atas da Sociedade de Biologia do Rio de Janeiro, v. 26, p. 37-40, 1986.

SÁO ClEMENTE, S. C. Plerocercos de cestóides da Ordem Trypanorhyncha em Corvina Micropogonias furnieri e sua importância na inspeção sanitária do pescado. Arquivos Fluminenses de Medicina Veterinária, v. 2, n. 3, p. 82-83, 1987.

SÃO CLEMENTE, S. C.; COELHO, M. R. T.; SERRA-FREIRE, N. M. Cestóides parasitos de Bagre Netuma barba (Lacépede, 1803) pescados no litoral do Rio de Janeiro e comercializados para consumo humano. Arquivos da Universidade Federal Rural do Rio de Janeiro, v. 14, n. 1, p. 27-34, 1991.

SÃO CLEMENTE, S. C. et al. Trypanorhyncha plerocerci in fish of commercial importance in Brazil. Parasilologia al Dia, v. 17, n. 1/2, p. 51-53, 1993.

SÃO CLEMENTE, S. C.; LIMA, F. C.; UCHOA, C. M. A. Parasitos de Balistes vetula e sua importância na inspeção do pescado. Revista Brasileira de Ciência Veterinária, v. 2, n. 2, p. 39-41, 1995.

SÃO ClEMENTE, S. C.; SILVA, C. M.; GOTTSCHALK, S. Prevalência e intensidade de infecção de cestóides Trypanorhyncha em Anchovas, Pomatomus saltatrix (L.) do litoral do Rio de Janeiro, Brasil. Parasilologia al Dia, v. 21, n. 1/2, p. 54-57, 1997.

SÃO CLEMENTE, S. C. et al. Cestóides Trypanorhyncha parasitos de Congro-rosa, Genypterus brasiliensis Regan, 1903 comercializados nos municípios de Niterói e Rio de Janeiro, Brasil. Revista Brasileira de Parasitologia Veterinária, v. 13, n. 3, p. 97-102, 2004.

SÃO CLEMENTE, S. C. et al. Cestóides Trypanorhyncha parasitos de peixe sapo-pescador, Lophius gastrophysus Miranda-Ribeiro, 1915 comercializados no estado do Rio de Janeiro, Brasil. Revista Brasileira de Parasitologia Veterinária, v. 16, n. 1, p. 37-42, 2007.

SILVA, C. M.; SÃO CLEMENTE, S. C. Nematóides da Família Anisakidae e cestóides da Ordem Trypanorhyncha em filés de dourado (Coryphaena hippurus) e ariocó (Lutjanus synagris) e sua importância na inspeção de pescado. Higiene Alimentar, v. 15, n. 80/81, p. 75-79, 2001.

THATCHER, V. E.; BRITES NETO, J. Diagnóstico, Prevenção e Tratamento das Enfermidades de Peixes Neotropicais de Água Doce. Revista Brasileira de Medicina Veterinária, v. 16, n. 3, p. 111-128, 1994. 